\title{
APPLICATION OF PETRI NETS METHODOLOGY FOR THE EXAMINATION OF THE BIO(PHYSICO)CHEMICAL PARAMETERS OF MITOCHONDRIA
}

\author{
FUNCTIONING
}

Hanna V. Danylovych *, Alexander Yu. Chunikhin, Yuriy V. Danylovych, Sergiy O. Kosterin

Palladin Institute of Biochemistry, National Academy of Sciences of Ukraine, Kyiv, Ukraine

*corresponding author: Danylovych Hanna, e-mail: danylovychanna@ukr.net

A mathematical model is developed using Petri net methodology that is able to predict simultaneous changes in bio(physical)chemical parameters of mitochondria, namely hydrodynamic diameter, fluorescence of NADH, DCF fluorescence signal depending on the time of action and concentration of sodium azide and predict these changes for unknown $\mathrm{NaN}_{3}$ concentration. The model is created for real experimental conditions, it combines functional changes of mitochondria with a structural representation of learned processes.

Keywords: Petri nets, mathematical modeling, mitochondria, electronic transport chain, nitric oxide.

\section{INTRODUCTION}

Biochemistry and molecular physiology of mitochondria, which combines the processes of oxygen consumption, oxidative phosphorylation, catabolism of lipids, biosynthesis of heme, maintenance of $\mathrm{Ca}^{2+}$ homeostasis, production of reactive oxygen and nitrogen species, apoptosis, etc. are the priority directions of modern biological science [1-3]. Further elucidation of the electronic transport chain functioning mechanisms and ways of 
breaking mitochondrial bioenergetics is important for understanding the causes and effects of mitochondrial dysfunction, which is the basis of the smooth muscles contractile function pathology. In particular, the generation of ATP and the functioning of low-affinity and highcapasitive $\mathrm{Ca}^{2+}$ uniporter significantly affects the path of $\mathrm{Ca}^{2+}$ signaling in myocytes and is an important factor in reducing the concentration of this cation in the myoplasm after the $\mathrm{Ca}^{2+}$ transient [4-5]. This also applies to the mitochondria of the uterus smooth muscle, where the electrochemical gradient dissipation of the internal membrane leads to a generalized increase in the myoplasm concentration of $\mathrm{Ca}^{2+}$ [6]. Inhibitors of some complexes of the respiratory chain are widely used in order to study thise.

Biomarkers of mitochondria functional activity are parameters such as the endogenous fluorescence signal from adenine nucleotides (NADH), the volume of mitochondria (hydrodynamic diameter), the intensity of reactive oxygen species production (DCF fluorescence), the efficiency of the $\mathrm{Ca}^{2+}$ accumulation, etc [4, 7-11]. The possibility of simultaneous simulation of these processes is important for understanding the functioning of mitochondria as a holistic system and will enable to predict the consequences of the violation of certain electronic transport chain components for bioenergy, $\mathrm{Ca}^{2+}$ homeostasis and programmed cell death. In particular, it seems advisable to construct a model based on data from the known inhibitor of the $I V$ complex of the respiratory chain and the "indirect NO donor " [12] sodium azide action on the bio(physical)chemical parameters of the mitochondria. The actuality of this work is due to the widespread use of $\mathrm{NaN}_{3}$ as a tool for inhibiting oxidative phosphorylation and transport functions of mitochondria, as well as the possibility of endogenous synthesis of NO in these subcellular structures.

We are developing a simulation model that links changes in the functioning of the electronic transport chain, the production of reactive oxygen species, the endogenous 
fluorescence of adenine nucleotides, and mitochondria swelling for the formalization and generalization of experimental data, in order to carry out the predictive function, and also to find the correspondence between the theoretical predictions and real results. We use the methodology of hybrid functional Petri nets as a modeling tool. The advantages of hybrid functional Petri nets as a modeling method include the following [13]: (1) capability to structurally represent the states of the modeled system and the processes occurring in the system; (2) quantitative modeling of three types of states and processes simultaneously, namely discrete, continuous, and associative (forming); (3) possibility to consider the activating, inhibiting, and catalytic effects by the means of a special type of bonds.

The aim of the paper was to use Petri nets to create an imitation model for simultaneous changes in the fluorescence of endogenous $\mathrm{NADH}$, the characteristic size of mitochondria and the generation of reactive oxygen species in real experimental conditions, which would combine functional changes with the structural representation of these processes.

\section{MATERIALS AND METHODS}

Experiments were performed on white wild-type nonpregnant rats weighing 150-180 g. All manipulations with animals were carried out according to the European Convention for the Protection of Vertebrate Animals used for Experimental and other Scientific Purposes, and the Law of Ukraine "On protection of animals from cruelty". Rats were anesthetized by diethyl ether inhalation and decapitated.

Isolation of mitochondria from the smooth muscle of the uterus (myometrium) of non-pregnant rats. The mitochondrial fraction was isolated from the myometrium of nonpregnant rats using differentiation centrifugation, as described by Kosterin and coworkers [14]. For the duration of the experiment, the isolated mitochondrial fraction was kept on ice. 
bioRxiv preprint doi: https://doi.org/10.1101/2020.01.22.915074; this version posted January 23,2020 . The copyright holder for this preprint (which was not certified by peer review) is the author/funder, who has granted bioRxiv a license to display the preprint in perpetuity. It is made available under aCC-BY-NC-ND 4.0 International license.

The protein content of the mitochondrial fraction was determined by a standard procedure by Bradford [15]. The total protein content in the mitochondrial fraction was $2 \mathrm{mg} / \mathrm{mL}$.

Registration of DCF-fluorescence in mitochondria. The loading of mitochondria by reactive oxygen species sensitive DCF-DA fluorescence probe at a concentration of $25 \mu \mathrm{M}$ was performed in a medium containing $10 \mathrm{mM}$ Hepes $\left(\mathrm{pH} 7.4,37^{\circ} \mathrm{C}\right), 250 \mathrm{mM}$ sucrose, $0.1 \%$ bovine serum albumin for $30 \mathrm{~min}$ at $24^{\circ} \mathrm{C}$. The Pluronic F-127 dye was added $(0.02 \%)$ to improve the loading process. DCF-fluorescense in isolated mitochondria was studied using the flow cytometry method on the COULTER EPICS XL ${ }^{\mathrm{TM}}$ (Beckman Coulter, USA) equipped with an $\operatorname{argon} \operatorname{laser}\left(\lambda_{\mathrm{ex}}=488 \mathrm{~nm}, \lambda_{\mathrm{fl}}=525 \mathrm{~nm}\right.$ (Fl1 channel). The reaction was initiated by adding aliquots $(20 \mu \mathrm{l})$ of $5 \mathrm{mM}$ pyruvate $+5 \mathrm{mM}$ succinate. Incubation time 35 min. Sodium azide were added for $15 \mathrm{~min}$. The protein content in the aliquot of the mitochondria fraction was $20-25 \mu \mathrm{g}$.

Estimation of mitochondrial hydrodynamic diameter. To assess changes in the mitochondrial volume, we used the Dynamic light scattering method, which allowed us to determine their sizes (average hydrodynamic diameter). The volume of the particles in suspension was analyzed using the correlation spectrometer ZetaSizer-3 (Malvern Instruments, UK) equipped with He-Ne laser LGN-111 (P=25 mW, $\lambda=633 \mathrm{~nm})$. Its operation principle is based on the analysis of time-dependent fluctuations in the scattering intensity when a laser ray passes through a medium with the mitochondria. The temporal intensity changes are converted into the mean translational diffusion coefficient (D) [16]. The translational diffusion coefficient is related to the duration of the correlation $\tau_{\mathrm{c}}$ with the following ratio:

$$
D q^{2}=1 / \tau_{c}
$$

The wave vector of the concentration fluctuations $(q)$ is described by the following expression: 


$$
q=\frac{4 \pi n}{\lambda_{o}} \sin \left(\frac{\theta}{2}\right)
$$

where $n$ is the refractive index of the medium (liquid), $\lambda_{0}$ is the wavelength, and $\theta$ is the scattering angle.

Using the Stokes-Einstein equation that connects the particle size, the translational diffusion coefficient, and the viscosity of the liquid, we could calculate the size (diameter) $\mathrm{d}(\mathrm{H})$ of the spherical particles as follows [16]:

$$
d(H)=\frac{k_{B} \cdot T}{3 \pi \eta D}
$$

where $k_{B}$ is the Boltzmann constant; $T$ is the absolute temperature, $\mathrm{K} ; \eta$ is the shear viscosity of the medium in which the particles are suspended; and $D$ is the translational diffusion coefficient.

The recording and statistical processing of the changes in the scattering intensity in the mitochondria water suspension $(n=1.33)$ were performed 10 times for $10 \mathrm{~min}$ at $+22^{\circ} \mathrm{C}$, at a scattering angle of $90^{\circ}$. The obtained results were processed using the PCS-Size mode v1.61 software.

The incubation medium consisted of $20 \mathrm{mM}$ Hepes $\left(\mathrm{pH} 7.4,37^{\circ} \mathrm{C}\right) 120 \mathrm{mM} \mathrm{KCl}, 2$ $\mathrm{mM}$ potassium-phosphate buffer $\left(\mathrm{pH} 7.4,37^{\circ} \mathrm{C}\right), 5 \mathrm{mM}$ pyruvate, and $5 \mathrm{mM}$ succinate.

Detection of NADH fluorescence in mitochondria using spectrofluorometry. The relative values of NADH intrinsic fluorescence were determined with Quanta Master 40 PTI (Canada) using the FelixGX 4.1.0.3096 software. The detection was conducted in $2 \mathrm{~mL}$ of the following medium: $20 \mathrm{mM}$ Hepes (pH 7.4 at $37^{\circ} \mathrm{C}$ ), $2 \mathrm{mM} \mathrm{K}{ }^{+}$-phosphate buffer (pH 7.4 at $\left.37^{\circ} \mathrm{C}\right), 120 \mathrm{mM} \mathrm{KCl}, 5 \mathrm{mM}$ pyruvate, and $5 \mathrm{mM}$ succinate. The protein concentration in the sample was $50 \mu \mathrm{g} / \mathrm{mL}$.

Simulation of DCF-fluorescence, mitochondrial swelling and changes in NADH fluorescence. For the simulation, we chose the Cell Illustrator v.3 software (Human Genome 
Center, University of Tokyo, Japan), the basis of which is a hybrid functional Petri net. A Petri net is a directed bipartite graph with two types of nodes (Table 1): places and transitions, which are connected by arcs, reflecting the structure of the net. Places usually characterize the objects, elements, and resources of the modeled system; transitions are the events that occur in the system and the logical conditions of their implementation [17].

Table 1. Main structural elements of the hybrid functional Petri nets (see the main text for the explanation)

\begin{tabular}{|c|c|c|c|c|}
\hline Type & Places & Transitions & Label & Arcs \\
\hline \multirow[t]{2}{*}{ Discrete } & & & Normal & threshold \\
\hline & Discrete place & Discrete transition & & Normal are \\
\hline \multirow[t]{2}{*}{ Continuous } & real number & $\square_{\text {rate }}$ & Test & - threshold \\
\hline & Continuous place & Continuous transition & & Test are \\
\hline \multirow[t]{2}{*}{ Generic } & $P_{\text {any types }}$ & בany operation & Inhibitory & threshold \\
\hline & Generic place & Generic transition & & Inhibitory are \\
\hline
\end{tabular}

Assessment of the content of ionized calcium in the mitochondria. Loading of the mitochondria by the probe Fluo-4 AM at a concentration of $2 \mu \mathrm{M}$ was carried out in the medium that contained $(\mathrm{mM})$ : Hepes $-10\left(\mathrm{pH} 7.4,37^{\circ} \mathrm{C}\right)$, sucrose -250 , and $0.1 \%$ bovine serum albumin for $30 \mathrm{~min}$ at $37^{\circ} \mathrm{C}$. To improve the process, the dye was mixed with Pluronic F-127 (0.02\%). The relative values of $\mathrm{Ca}^{2+}$ content in the matrix of mitochondria, loaded with Fluo-4 AM $\left(\lambda_{\mathrm{ex}}=490 \mathrm{~nm}, \lambda_{\mathrm{fl}}=520 \mathrm{~nm}\right)$ was investigated using the fluorometric method on spectrofluorometer Quanta Master PTI 40 (Canada) with software FelixGX 4.1.0.3096. The medium, from which energy-dependent accumulation of $\mathrm{Ca}^{2+}$ was carried out by the

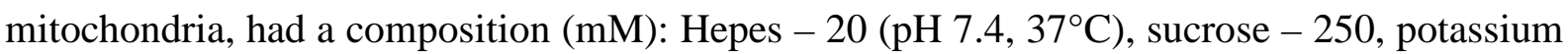


phosphate buffer - $2\left(\mathrm{pH} 7.4,37^{\circ} \mathrm{C}\right), \mathrm{MgCl}_{2}-3$, ATP -3 , sodium succinate - 5, concentration of $\mathrm{Ca}^{2+}-80 \mu \mathrm{M}[18]$.

In the work the following reagents were used: Hepes, glucose, sucrose, sodium succinate, sodium pyruvate, bovine serum albumin, ATP, $\mathrm{NaN}_{3}$, Pluronic F-27, DCF-DA (2',7'-dichlorodihydrofluorescein diacetate), EGTA, $\mathrm{CaCl}_{2}, \mathrm{~A}-23187$ (Sigma, USA); Fluo-4 AM (Invitrogen, USA). Any other reagents are produced in Ukraine.

The solutions were prepared on bidistilled water, which had a specific electrical conductivity of not more than $2.0 \mu \mathrm{cm} / \mathrm{cm}$. The electrical conductivity of the water was recorded using a conductometer OK-102/1 (Hungary).

\section{RESULTS AND DESCUSSION}

We have developed a simulation model (Fig. 1) that connects changes in the endogenous NADH fluorescence (Fig. 2), production of reactive oxygen species (Fig. 3) and mitochondria swelling (Fig. 4) for the formalization of experimental data in this work, in order to carry out the predictive function, and also to find the correspondence between the theoretical predictions and real results. We have used the "indirect donor" of NO sodium azide as biologically active compound that cause changes in the corresponding bio(physical)chemical parameters of the mitochondria (Fig. 3,4).

It was established, that $\mathrm{NaN}_{3}$ increased the mitochondria hydrodynamic diameter (causing swelling) and amplified their DCF fluorescence (it stimulated the formation of reactive oxygen forms), depending from its concentration (1-3-5-7 $\mathrm{mM})$ and time (0-15 $\mathrm{min})$, see Fig. 3, 4. Inhibition of the respiratory chain $I V$ complex results to the slowing down of the mitochondria functioning, the reduction of the electrical and chemical potential on the internal membrane $(\Delta \mathrm{pH})$, the opening of the permeability transient pore (MPTP) and the mitochondria swelling [19-23]. The accumulation in the electron transport chain of the semi- 
reduction components, especially in Q-cycle, leads to an increase in the process of oneelectron oxygen reduction [24]. Thus, the generation of superoxide anion and other reactive oxygen species will be intensified. Simultaneously, the chemical decomposition of sodium azide with the formation of nitrogen oxides will be accompanied by an increase in the generation of peroxynitrite, which contributes to DCF fluorescence too. Strengthening under these conditions the content of reactive nitrogen and oxygen species in mitochondria will further reduce the functional activity of the electron transport chain and matrix enzymes, especially those containing iron-sulfur centers and metal proteins [25-27]. The latter will result in the processes of the mitochondrial dysfunction, collapse of bioenergetics up to cell death $[21,23,28]$. Using Petri hybrid functional nets, we simulated the effect of sodium azide, depending on the time and concentration, on the hydrodynamic diameter and DCF fluorescence of the myometrium isolated mitochondria.

The components of the mitochondria incubation medium and the effects of sodium azide on their bio(physical)chemical parameters were taken into account in the simulation. We operated the following experimental facts in the process of the model creation: (1) succinate and pyruvate are added to the mitochondria incubation medium for their energized; (2) - sodium azide, as an "indirect NO donor ", has an inhibitory effect on the electronic transport chain as a whole, but the greatest effect on the morpho-functional parameters of the organelles has inhibition of the $I, I I I$ and $I V$ complexes (3) - inhibition of the electron transport chain activity results in the intensification of the reactive oxygen forms production in the mitochondria and, consequently, DCF fluorescence (direct inhibition of the $I V$ complex within the framework of the simulation model); (4) - inhibition of the electron transport chain by sodium azide leads to an increase in the hydrodynamic diameter of the mitochondria: the osmotic balance between the matrix and the non-mitochondrial medium is broken due to the activation of the permeability transition pore with the subsequent $\mathrm{H}_{2} \mathrm{O}$ entrance to the matrix 
bioRxiv preprint doi: https://doi.org/10.1101/2020.01.22.915074; this version posted January $23,2020$. The copyright holder for this preprint (which was not certified by peer review) is the author/funder, who has granted bioRxiv a license to display the preprint in perpetuity. It is made available under aCC-BY-NC-ND 4.0 International license.

and swelling of the mitochondria (this is the result of inhibition of $I, I I I$ and $I V$ complexes and depolarization of the internal mitochondrial membrane in the framework of the simulation model).

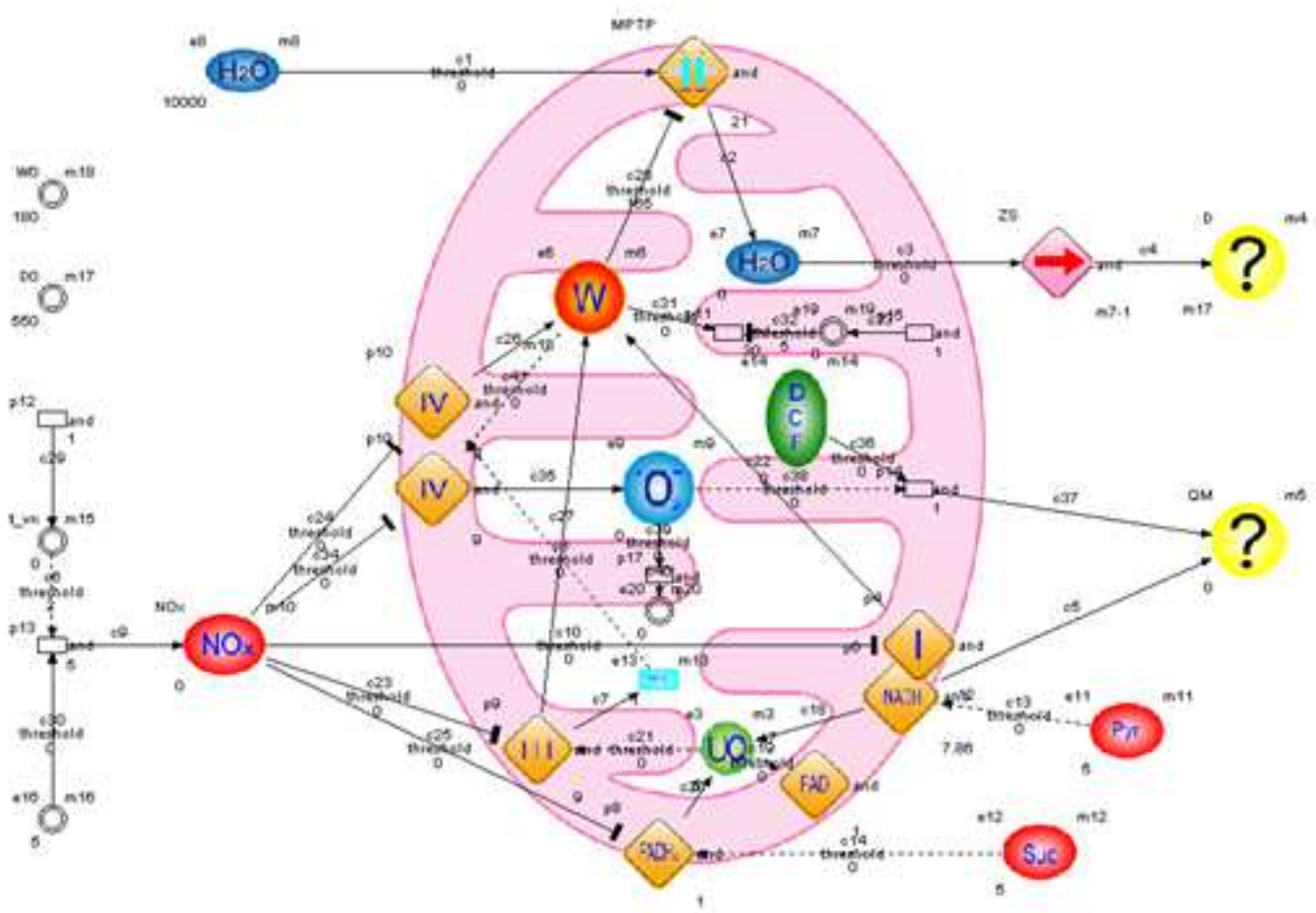

Fig. 1. Structure of hybrid functional Petri nets, which simulated the sodium azide effect on the changes in the hydrodynamic diameter and the NADH, DCF fluorescence of the myometrium mitochondria

Symbols in the scheme: $\mathrm{NO}_{\mathrm{x}}$-nitrocompounds, particularly sodium azide; Suc— sodium succinate; Pyr-sodium piruvate; W-electric potential of the inner mitochondrial membrane; I, II, III, and IV as well as $\mathrm{FADH}_{2}, \mathrm{FAD}$, and NADH (inscribed in a rhombus in the mitochondrial membrane) - complexes of the electron transport chain and the appropriate cofactors; $\mathrm{O}_{2}{ }^{--}$- superoxide anion; DCF - DCF-DA fluorescence probe; UQ-ubiquinone; cyt $\mathrm{C}$-cytochrome $\mathrm{C}$; symbol -| |- inside a rhombus-cyclosporine-sensitive permeability transition pore, MPTP; and arrows: $\rightarrow-$ activation of the process and $\mathrm{T}^{-}$inhibition of the process 
Structure of hybrid functional Petri nets, which simulated the sodium azide effect on the changes in the hydrodynamic diameter, NADH and DCF fluorescence of the myometrium mitochondria is presented on scheme (Fig. 1). Symbols in the scheme are explained on the caption. Some particular specifications are listed below. The place m15 is a timer that controls the time of the inhibitor insertions in the amount determined by the place m16. Before the insertion of a specific inhibitor of electron transport chain, the mitochondrial hydrodynamic diameter, measured by Zeta-sizer (transition ZS and place m4) practically does not change. The fluorescent response of NADH/DCF is registered by the spectrofluorimeter QM (place m5) and corresponds to the "control" curves (Fig. 2, 3).

The insertion of sodium azide $\left(\mathrm{NO}_{\mathrm{x}}\right.$, Fig. 1) breaks the work of electron transport chain that decreases the electric potential of the inner mitochondrial membrane $\mathrm{W}$ (place m6). It causes an activation of the mitochondrial permeability transition pore (MPTP), $\mathrm{H}_{2} \mathrm{O}$ transport into the matrix (place $\mathrm{m} 7$ ), and an increase in the mitochondrial hydrodynamic diameter. The fluorescence response changes are simulated by the NADH and DCF transitions with velocities in accordance with equations $(5,7)$ as described below.

Through the modeling, we obtained mathematical equations that formalized the process of mitochondria swelling and the changes in the NADH/DCF fluorescence in the medium supplemented with sodium azide. In particular, these equations adequately described the time characteristics of the mitochondria swelling (Fig. 4), changing of the NADH/DCF fluorescence (Fig. 2, 3) simultaneously. The permeability of MPTP and the intensity of the $\mathrm{NADH} / \mathrm{DCF}$ fluorescent response are the time derivatives from the corresponding dependencies.

The substrate of pyruvate dehydrogenase complex $(5 \mathrm{mM}$ pyruvate $)$ that produced $\mathrm{NADH}$ for electron transport chain, and $5 \mathrm{mM}$ succinate, a substrate of FAD-dependent succinate dehydrogenase was into incubation medium in order to produce the energized state 
of mitochondria [29]. It has been shown that NADH fluorescence decreased in time in the presence of respiratory substrates, which indicates increase in $\mathrm{NAD}^{+}$content resulting from functioning of NADH-ubiquinone oxidoreductase of electron transport chain (Fig. 1). We have used experimental results from reduce the auto-fluorescence of NADH in isolated mitochondria over time under the condition of the electron transport chain functioning for modeling in this study. The time dependence of the average change in the fluorescent response of NADH (autofluorescence) was approximated by polynomials as follows:

$$
\operatorname{Fn}(\mathrm{NADH})=-0,07 \mathrm{t}^{3}+2,54 \mathrm{t}^{2}-32,72 \mathrm{t}-12,19
$$

The intensity of the NADH fluorescent response:

$\mathrm{dFn} / \mathrm{dt}=-0,21 \mathrm{t}^{2}+5,1 \mathrm{t}-32,72$

\section{Time, $\min$}

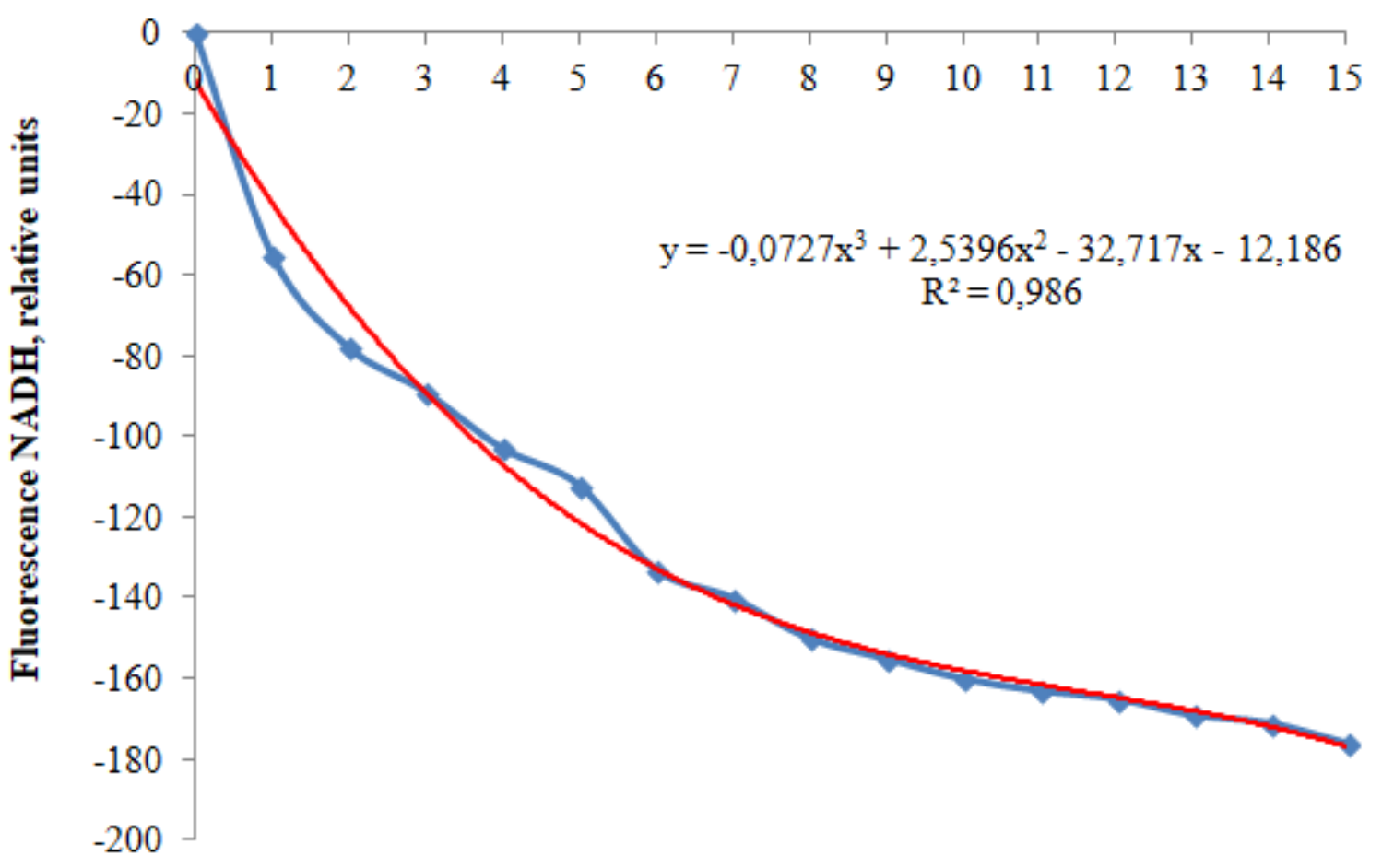

Fig. 2. Changes in fluorescence of NADH in the isolated mitochondria from the myometrium cells. These data represent a typical experiment 
The concentration-time dependencies of the DCF fluorescence can be approximated by polynomials as:

$$
F n(D C F)=\left(-0,099 c^{2}+2,57 c+1,854\right) t+2,506 c+990,7
$$

where $\mathrm{c}$ - concentration of sodium azide, $\mathrm{mM}, \mathrm{t}$ - time, min.

The intensity of the DCF fluorescence:

$$
d F n / d t=-0,099 c^{2}+2,57 c+1,854
$$

The obtained expressions make it possible to predict the time dependence of DCF fluorescence, for example, for $10 \mathrm{mM}$ concentration of sodium azide (Fig. 3):

$$
F n(D C F)=17,65 t+1015,8
$$

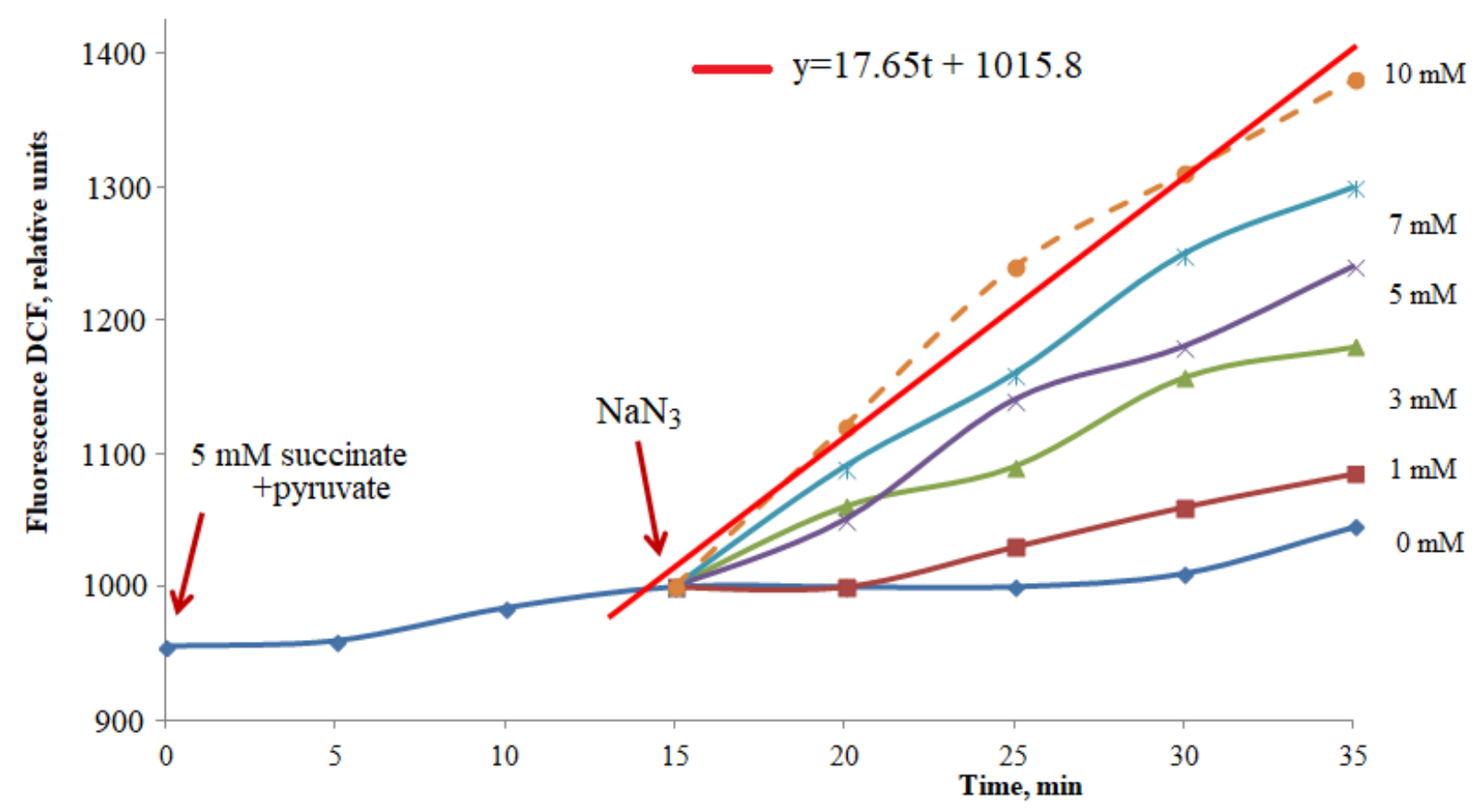

Fig. 3. The concentration-time dependencies of the DCF fluorescence in the isolated mitochondria. The straight red line is theoretically calculated according to equation (8) for 10 $\mathrm{mM}$ sodium azide; dotted brawn line - experimental obtained curve for $10 \mathrm{mM}$ sodium azide. These data represent a typical experiment 
bioRxiv preprint doi: https://doi.org/10.1101/2020.01.22.915074; this version posted January 23,2020 . The copyright holder for this preprint (which was not certified by peer review) is the author/funder, who has granted bioRxiv a license to display the preprint in perpetuity. It is made available under aCC-BY-NC-ND 4.0 International license.

According to results for $1-7 \mathrm{mM} \mathrm{NaN}_{3}$ (Fig. 4) dynamics of the concentrationdependent changes in the average values of mitochondria hydrodynamic diameter during swelling can be approximated as:

$\Delta D=(0,7 c+31) \ln (t)+16,6 c-39$

where $\mathrm{c}-$ concentration of sodium azide, $\mathrm{mM}, \mathrm{t}-$ time, $\min$.

So, the permeability of mPTP is described as:

$d(\Delta D) / d t=(0,7 c+31) / t$

It is possible to predict theoretically the course of the corresponding curves for unknown sodium azide concentrations based on this equation. Fig. 4 shows an example of such a curve for $10 \mathrm{mM}$ sodium azide.

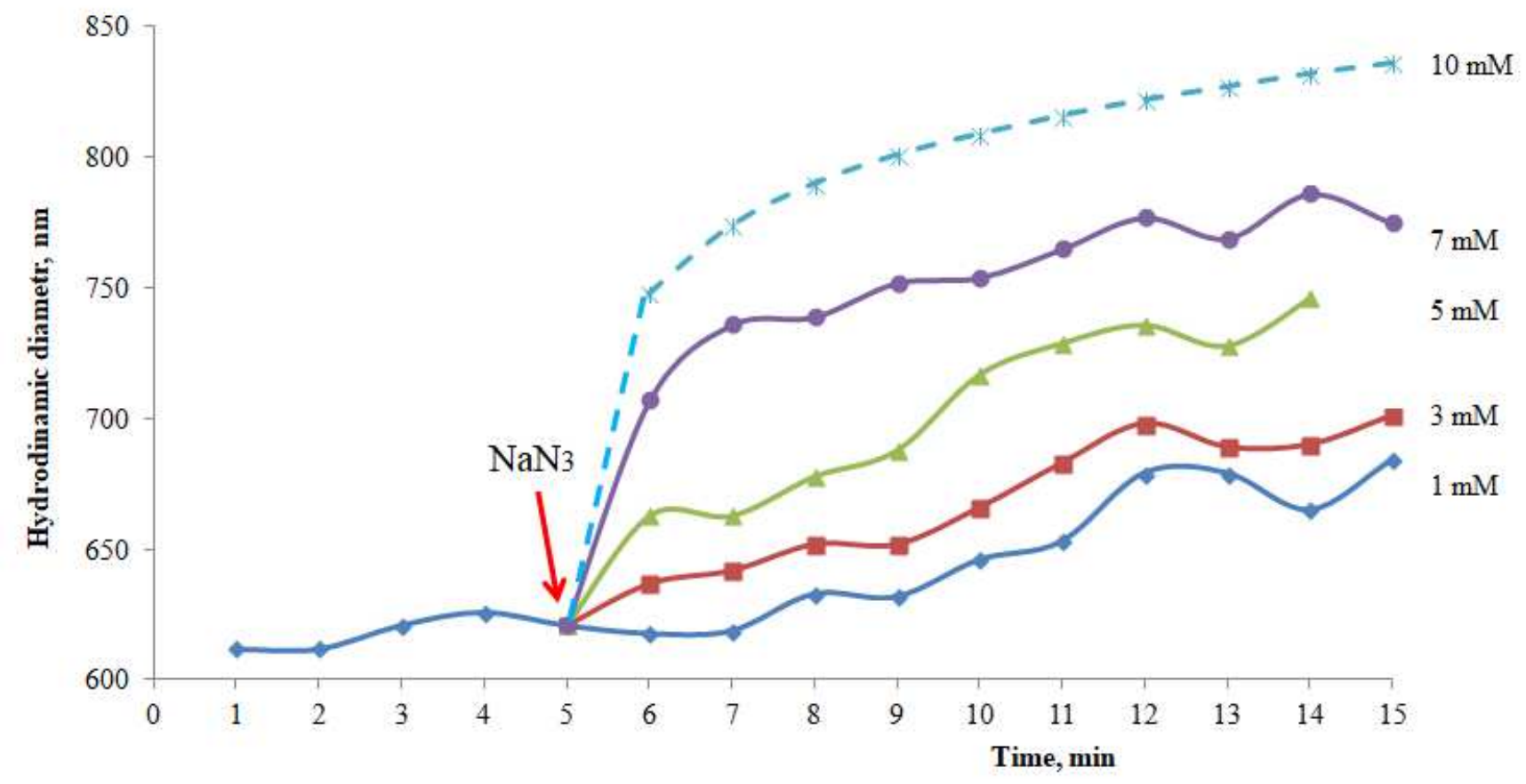

Fig. 4. The values of mitochondria hydrodynamic diameter during swelling on $\mathrm{NaN}_{3}$ action. The curve for $10 \mathrm{mM}$ sodium azide is theoretically calculated according to equation (9). These data represent a typical experiment 
Our model enabled a simultaneous prediction of the changes in the organelle $\mathrm{NADH} / \mathrm{DCF}$ fluorescence and their hydrodynamic diameter in time, which enabled us to significantly optimize the time of the experimental procedures, the consumption of reagents, and the use of laboratory animals. Moreover, it allowed us to analyze the dynamics of the processes and to compare the modeling results with the actual observations, considering the changes in the abovementioned parameters (the composition of the incubation medium and the presence of the activators/inhibitors).

Sodium azide is known to degrade in water solutions, producing hydrazoic acid, hydroxylamine, and, possibly, nitrogen oxides, which act as reactive nitrogen species in biological systems [12]. In our previous experiments sodium azide (5 mM) caused more pronounced decrease in NADH fluorescence than in control, and also increase in $\mathrm{FADH}_{2}$ contents, which may indicate blocking of electron transfer from succinate to ubiquinone [30]. We have attributed the decreased $\mathrm{NADH}$ and FAD levels under nitrocompounds to drop in activity of enzymes of citric acid cycle due to inhibition of electron transport chain in mitochondrial membrane. Thus, we investigated with flow cytometry the effect of the sodium azide on matrix content of $\mathrm{Ca}$ ions in mitochondria to confirm the effect of $\mathrm{NO}_{\mathrm{x}}$ on the mitochondria functional activity (Fig. 5). Addition of exogenous $\mathrm{Ca}^{2+}$ to mitochondria suspension was associated with the increase in fluorescence of Fluo- 4 that had been loaded into them in advance, which indicates increased matrix $\mathrm{Ca}^{2+}$ concentration. The cation was accumulated in the presence of $\mathrm{Mg}_{-} \mathrm{ATP}^{2-}$ and succinate for $5 \mathrm{~min}$, at which moment the stable level of $\mathrm{Ca}^{2+}$ accumulation was achieved (Fig 5, column 1). We ascertained the barrier function of mitochondrial inner membrane towards $\mathrm{Ca}$ ions by addition of $\mathrm{A} 23187 \mathrm{Ca}^{2+}$ ionophore to suspension. This was associated with rapid release of the accumulated cation (Fig 5, column 2). The sodium azide caused efficient release of accumulated $\mathrm{Ca}^{2+}$ from mitochondria (Fig 5, column 3). The deenergizing effect of sodium azide on mitochondria has 
been reliable established. The slight increase in ionized $\mathrm{Ca}$ in the matrix was observed, as well as a significant decrease in $\mathrm{Ca}$ accumulation in comparison with the control, under conditions of mitochondrial pre-incubation with sodium azide (depolarization of the internal membrane) (Fig. 5, column 4 and 5 respectively).

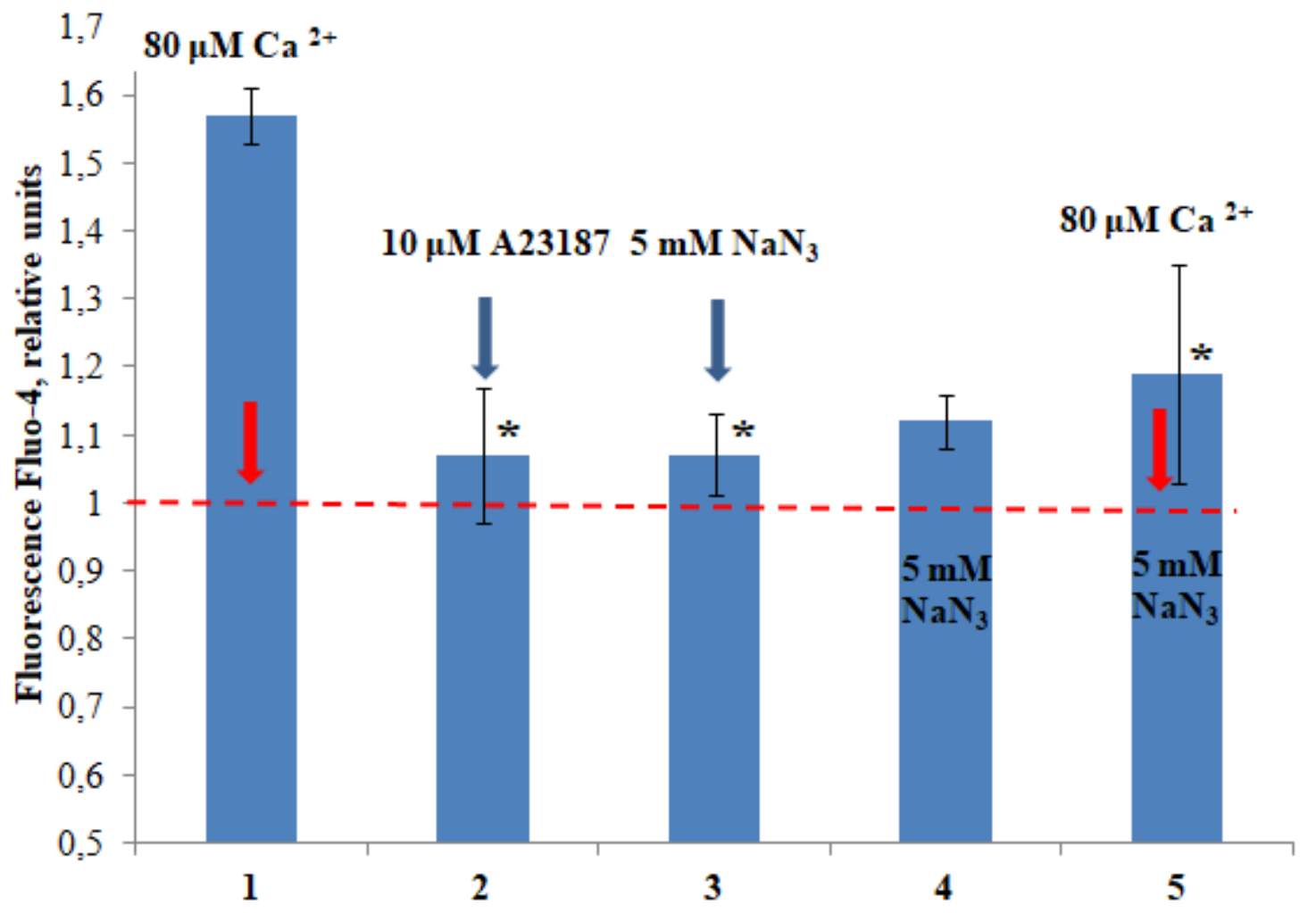

Fig. 5. Effects of $\mathrm{Ca}^{2+}$-ionophore and sodium azide on matrix $\mathrm{Ca}^{2+}$ content. The cation had been accumulated in energy-dependent manner. Endogenous ionized $\mathrm{Ca}$ - “1”, starting level. The data are presented as mean $\pm \mathrm{SEM}, *-\mathrm{P} \leq 0.05, \mathrm{n}=5$

Thus, using the Petri nets, the simulation model for simultaneous changes in the fluorescence of endogenous $\mathrm{NADH}$, the characteristic size of mitochondria, and the generation of reactive oxygen species under real experimental conditions has been created, which combines functional changes with the structural representation of these processes. The simulation results in mathematical equations that formalize simultaneous processes of mitochondrial swelling and DCF fluorescence in a medium with $\mathrm{NaN}_{3}$. The de-energizing 
effect of sodium azide on mitochondria results in disturbance in their functioning, namely the ability to effectively accumulate and maintain $\mathrm{Ca}^{2+}$ in their matrix.

\section{CONCLUSIONS}

The simulation results in mathematical equations that formalize simultaneous processes of mitochondrial swelling, changes in NADH and DCF fluorescence in a medium in which $\mathrm{NaN}_{3}$ is presented. These equations are able to adequately describe the time and concentration characteristics of these processes, as well as predict the intensity of their occurrence. In particular, the response of mitochondria over time to the action of $\mathrm{NaN}_{3}$ in a concentration, that was not used in the experiment, was predicted. The calculated values of the studied bio(physical)chemical parameters were consistent with experimentally obtained data. Creation of an adequate model optimizes experimental procedures (time, costs of reagents and laboratory animals), allows analyzing the dynamics of processes and comparing experimental results with theoretical calculations provided that the composition of the incubation medium changes.

\section{REFERENCES}

1. Bernardi P, Rasola A. Calcium and cell death: the mitochondrial connection. Subcellular Biochemistry. 2007;45:481-506.

2. Graier WF, Frieden M, Malli R. Mitochondria and $\mathrm{Ca}^{2+}$ signaling: old quests, new functions. Eur J Physiol. 2007;455(3):375-96.

3. Orrenius S, Packer L, Cadenas E, editors. Mitochondrial signaling in health and disease. N.Y.: CRC Press, 2012. 493 p. 
4. Csordás G, Várnai P, Golenár T, Sheu S-S, Hajnóczky G. Calcium transport across the inner mitochondrial membrane: molecular mechanisms and pharmacology. Mol Cell Endocrinol. 2012;353(1-2):109-113.

5. Szabadkai G, Duchen MR. Mitochondria: The Hub of Cellular $\mathrm{Ca}^{2+}$ Signaling. Physiology. 2008;23(2):84-94.

6. Danylovych YuV, Karakhim SA, Danylovych GV, Kolomiets OV, Kosterin SO. Electrochemical potential of the inner mitochondrial membrane and $\mathrm{Ca}^{2+}$ homeostasis of myometrium cells. Ukr Biochem J. 2015;87(5):56-65.

7. Wang HW, Wei YH, Guo HW. Reduced nicotinamide adenine dinucleotide $(\mathrm{NADH})$ fluorescence for detection of cell death. Anticancer Agent Med Chem. 2009;9(9):1012-7.

8. Heikal AA . Intracellular coenzymes as natural biomarkers for metabolic activities and mitochondrial anomalies. Biomark Med. 2010;4(2):241-63.

9. Brocard JB, Rintoul GL, Reynolds IJ. New perspectives on mitochondrial morphology in cell function. Biol Cell. 2003;95:239-42.

10. Kaasik A, Safiulina D, Zharkovsky A, Veksler V. Regulation of mitochondrial matrix volume. Am J Physiol Cell Physiol. 2007;292:C157-C163.

11. Nowikovski K, Schweyen RJ, Bernardi P. Pathophysiology of mitochondrial volume homeostasis: potassium transport and permeability transition. Biochim Biophys Acta. 2009;1787(5):345-350.

12. Iakovenko IN, Zhirnov VV. Sodium azide as indirect nitric oxide donor: researches on the rat aorta isolated segments. Ukr Biokhim Zh. 2005;77(4):120123. (in Russian).

13. Wingender E., editor. Biological Petri Nets. IOSPress; 2011. 
14. Kosterin SA, Bratkova NF, Kursky MD. The role of sarcolemma and mitochondria in $\mathrm{Ca}^{2+}$-dependent control of myometrium relaxation. Biochemistry (Moscow). 1985;50:1350-61.

15. Bradford MM. A rapid and sensitive method for the quantitation of microgram quantities of protein utilizing the principle of protein-dye binding. Anal Biochem. $1976 ; 72: 248-254$.

16. Merkus H.G. Particle size measurements. Fundamentals, practice, quality. Springer; 2009.

17. Nagasaki M, Saito A, Doi A, Matsuno H, Miyano S. Foundations of Systems Biology. Using Cell Illustrator and Pathway Databases. Springer; 2009.

18. Kolomiets OV, Danylovych YuV, Danylovych GV, Kosterin SO. $\mathrm{Ca}^{2+}$ accumulation study in isolated smooth muscle mitochondria using Fluo-4 AM. Ukr Biokhim Zh. 2013;85(4):30-9. (in Ukraine).

19. Chang S, Lamm SH. Human health effects of sodium azide exposure: a literature review and analysis. Int J Toxicol. 2003;22(3):175-86.

20. Ji D, Kamalden T A, del Olmo-Aguado S, Osborne NN. Light- and sodium azideinduced death of RGC-5 cells in culture occurs via different mechanisms. Apoptosis. 2011;16(4):425-37.

21. Feissner RF, Skalska J, Gaum WE, Sheu SS. Crosstalk signaling between mitochondrial $\mathrm{Ca}^{2+}$ and ROS. Front Biosci. 2009;14:1197-218.

22. Santo-Domingo J, Wiederkehr A, De Marchi U. Modulation of the matrix redox signaling by mitochondrial $\mathrm{Ca}^{2+}$. World J Biol Chem. 2015;6(4):310-23.

23. Bernardi $\mathrm{P}$, von Stockum $\mathrm{S}$. The permeability transition pore as a $\mathrm{Ca}^{2+}$ release channel: New answers to an old question. Cell Calcium. 2012;52(1):22-7. 
24. Trumpower B.L. The protonmotive Q cycle. Energy transduction by coupling of proton translocation to electron transfer by the cytochrome bc1 complex. J Biol Chem. 1990;265(20):11409-12.

25. Bringold U, Ghafourifar P, Richter C. Peroxynitrite formed by mitochondrial NO synthase promotes mitochondrial $\mathrm{Ca}^{2+}$ release. Free Radic Biol Med. 2000;29:3438.

26. Gellerich FN, Gizatullina Z, Trumbeckaitec S, Nguyen HP, Pallas Th, Arandarcikaite O, Vielhaber S, Seppet E, Striggow F. The regulation of OXPHOS by extramitochondrial calcium. Biochim Biophys Acta. 2010; 1797: 1018-27.

27. Elfering SL, Haynes VL, Traaseth NJ, Ettl A, Giulivi C. Aspects, mechanism, and biological relevance of mitochondrial protein nitration sustained by mitochondrial nitric oxide synthase. Am J Physiol Heart Circ Physiol. 2004;286:H22-9.

28. Rasola A, Bernardi P. Mitochondrial permeability transition in $\mathrm{Ca}^{2+}$-dependent apoptosis and necrosis. Cell Calcium. 2011;50(3):222-33.

29. Chance B, Williams GR. Respiratory enzymes in oxidative phosphorylation. III. The steady state. J Biol Chem. 1955;217:409-27.

30. Danylovych H., Chunikhin A., Danylovych Yu., Kosterin S. Methodology of Petri networks for simultaneous evaluation of the impact of different modifiers on the fluorescence of nucleotides from electron transport chain in isolated mitochondria and on the process of swelling. $\mathrm{J}$ Biotech Comput Biol Bionanotech. 2018;99(1):37-48. 